\title{
A short communication about the article "Assessment of the length of sick leave in patients with ischemic heart disease"
}

\author{
Nausica Català Tella', Catalina Serna Arnaiz' ${ }^{1}$ Jordi Real Gatius ${ }^{2,3}$, Oriol Yuguero Torres ${ }^{4}$ and Leonardo Galván \\ Santiago ${ }^{5}$ \\ 'ABS Eixample, Institut Català de la Salut, Lleida, Spain \\ ${ }^{2}$ Institut Universitari d'Investigació en Atenció Primària Jordi Gol (IDIAP Jordi Gol), Lleida, Spain \\ ${ }^{3}$ Facultat de Medicina i Ciències de la Salut, Universitat Internacional de Catalunya, Sant Cugat, Barcelona), Spain \\ ${ }^{4}$ Servicio de Urgencias del Hospital Universitari Arnau de Vilanova, Lleida, Spain \\ ${ }^{5}$ Servicio de farmacia, Departament Català de la Salut, Lleida, Spain
}

Article Info

\section{Article Notes}

Received: November 6, 2017

Accepted: December 13, 2017

\section{*Correspondence:}

Dr. Nausica Català Tella, ABS Eixample, Institut Català de la Salut, Balmes avenue, 4, 25006, Lleida, Spain; Email: ncatala@hotmail.com.

(c) 2017 Català Tella N. This article is distributed under the terms of the Creative Commons Attribution 4.0 International License.

\section{Keywords}

Acute Coronary Syndrome

Ischemic Heart Disease

\section{Background}

Ischemic heart disease (IHD) is particularly important in our environment due to its high prevalence, the high consumption of resources it requires, and the associated mortality. The increase in its prevalence and advances in technology have established IHD as the disease with the greatest economic impact in the developed countries ${ }^{1,2}$. In recent decades, mortality rates after IHD have declined in developed countries, but IHD nonetheless remains the leading cause of death in men and the second most frequent in women ${ }^{1,3}$. Incapacity for work is one of the indirect costs of the disease, but this issue has not been widely studied ${ }^{1,2,4,5}$. Few authors have assessed the length of periods of sick leave due to IHD ${ }^{1,6-10}$ since the review of the topic conducted in 2004 by Perk and Alexanderson ${ }^{1,11}$. The study by Sicras-Mainar in 2009 quantified the cost per patient following an episode of Acute Coronary Syndrome (ACS) at 14,069 euros (87\% of which were direct costs and $13 \%$ lost productivity costs). The direct costs comprised primary care (20\%) and specialized care $(67 \%)$, with hospitalization costs accounting for $63 \%$ of the total ${ }^{1,4}$. Our aim is to determine the length of sick leave after an acute coronary syndrome, its costs, associated factors and to assess the use of antidepressants and/or anxiolytics ${ }^{1}$.

\section{Methods}

An observational study of a retrospective cohort of patients on sick leave due to ischemic heart disease in a health region of Catalonia, Spain, between 2008-2011, with follow-up until the first return to work, death, or end of the study (31/12/2012). Measurements: length of sick leave, sociodemographic variables and medical prescriptions. Data were obtained from the electronic medical record database of the primary care section (ECAP) of the Catalan Health Service and from the pharmacy billing databases run by CatSalut ${ }^{1}$.

The duration of sick leave was estimated by Kaplan-Meier survival analysis until the first medical discharge. The periods of sick leave were compared using the Log-Rank test, and at the multivariate level, hazards ratios were estimated by adjusting the Cox regression models. The assumption of proportional risks 
of the Cox models was evaluated using the Schofield residuals ${ }^{1}$.

Indirect costs were those relating to productivity loss (number of periods of sick leave and total days off work). They were quantified according to the minimum wage (Source: Spanish Institute of Statistics), considering the cost per day not worked at 54.65 euros $^{1,4}$. Indirect costs were estimated for the whole of the patients' follow-up period ${ }^{1}$.

\section{Results}

Four hundred and ninety-seven patients (mean age 53 years, 90.7\% male), diagnosed with acute myocardial infarction (60\%), angina pectoris (20.7\%) or chronic form of ischemic heart disease (19.1\%). Thirty-seven per cent of patients took anxiolytics the year after diagnosis and 15\% took antidepressants (Table 1). The average duration of sick leave was 177 days (95\% CI: 163-191 days). Patients diagnosed with acute myocardial infarction returned to work after a mean of 192 days, compared to 128 days in cases with angina pectoris (Fig.1). Patients who took antidepressants during the year after diagnosis returned to work after a mean of 240 days (Table 2). Table 3 shows the multivariate analysis of the hazards ratios for returning to work depending on the variables analysed. The mean work productivity loss was estimated to be 9,673 euros/person ${ }^{1}$.

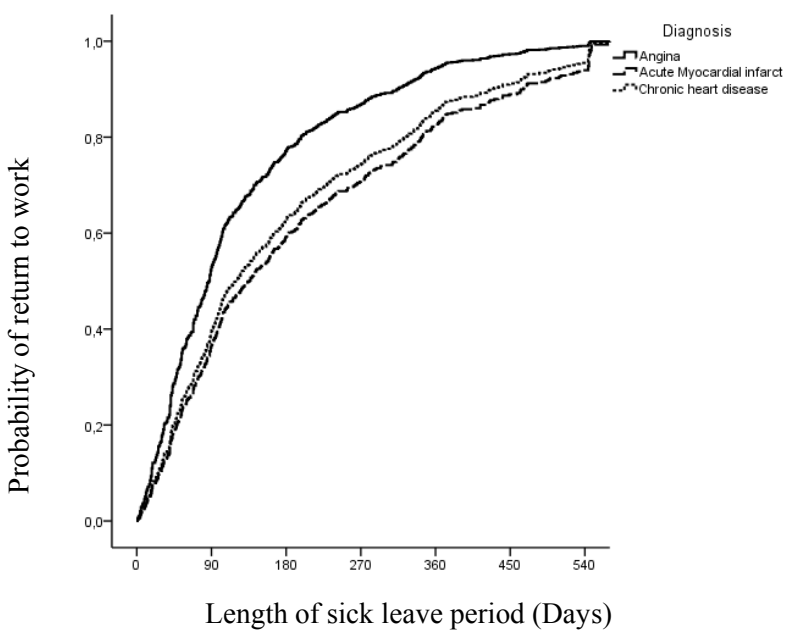

Figure 1 Probability of return to work in relation to the length of sick leave period

Table 1: Descriptive data of the variables analysed

\begin{tabular}{|c|c|c|}
\hline Variables & $\mathrm{N}$ & $\%$ \\
\hline \multicolumn{3}{|l|}{ Diagnostic code (CIE10) and description } \\
\hline Angina (120) & 103 & $20.70 \%$ \\
\hline Acute myocardial infarct (AMI) (I21) & 295 & $59.40 \%$ \\
\hline Complications following AMI (I23) & 1 & $0.20 \%$ \\
\hline Other acute ischemic heart disease (124) & 3 & $0.60 \%$ \\
\hline Chronic ischemic heart disease (125) & 95 & $19.10 \%$ \\
\hline \multicolumn{3}{|l|}{ Age group } \\
\hline$<=45$ years & 88 & $17.70 \%$ \\
\hline 46 to 55 & 195 & $39.20 \%$ \\
\hline$>=56$ & 214 & $43.10 \%$ \\
\hline \multicolumn{3}{|l|}{ Year of sick leave } \\
\hline 2008 & 142 & $28.60 \%$ \\
\hline 2009 & 117 & $23.50 \%$ \\
\hline 2010 & 112 & $22.50 \%$ \\
\hline 2011 & 126 & $25.40 \%$ \\
\hline \multicolumn{3}{|l|}{ Sex } \\
\hline Female & 48 & $9.70 \%$ \\
\hline Male & 449 & $90.30 \%$ \\
\hline \multicolumn{3}{|l|}{ Medication dispensed : Yes/No ATC code) } \\
\hline \multicolumn{3}{|l|}{ Prior sedative (N03AE N05BA-BB-BX-CD-CF-CM) } \\
\hline Previous & 136 & $27.4 \%$ \\
\hline Previous 6 months & 73 & $14.7 \%$ \\
\hline Previous 12 months & 95 & $19.1 \%$ \\
\hline Subsequent 12 months & 184 & $37.0 \%$ \\
\hline \multicolumn{3}{|l|}{ Antidepressants GT=N06A / N06B / N06C } \\
\hline Previous & 74 & $14.9 \%$ \\
\hline Previous 6 months & 30 & $6.0 \%$ \\
\hline Previous 12 months & 39 & $7.8 \%$ \\
\hline Subsequent 12 months & 76 & $15.3 \%$ \\
\hline
\end{tabular}


Table 2: Estimated time in days of the overall period of sick leave according to group

\begin{tabular}{|c|c|c|c|c|c|c|c|c|}
\hline & & & \multicolumn{2}{|c|}{$95 \% \mathrm{Cl}$} & \multirow{3}{*}{$\begin{array}{c}\text { Median } \\
116\end{array}$} & \multicolumn{2}{|c|}{$95 \% \mathrm{Cl}$} & p-value \\
\hline Variable & & Mean & $\begin{array}{l}\text { (Upper } \\
\text { limit - }\end{array}$ & $\begin{array}{r}\text { Lower } \\
\text { limit) }\end{array}$ & & $\begin{array}{l}\text { (Upper } \\
\text { limit - }\end{array}$ & \multicolumn{2}{|c|}{$\begin{array}{l}\text { Lower } \\
\text { limit) }\end{array}$} \\
\hline Global & & 177.3 & $(163.5-$ & 191.0) & & (98.6- & 133.4) & \\
\hline Diagnostic code (CIE10) and description & & & & & & & & 0.002 \\
\hline Angina (I20) & & 127.8 & (99.9- & 155.6) & 68 & $(46.6-$ & 89.4) & \\
\hline Acute Myocardial Infarction (I21) & & 191.8 & (174.1- & 209.5) & 131 & (102.1- & 159.9) & \\
\hline \multicolumn{2}{|l|}{ Other acute ischemic heart disease (I24) } & 143.0 & $(47.8-$ & 238.2) & 187 & $(0.0-$ & 412.6) & \\
\hline \multicolumn{2}{|l|}{ Chronic ischemic heart disease (I25) } & 187.7 & $(154.5-$ & 220.9) & 137 & $(105.7-$ & 168.3) & \\
\hline Age group (in years) & & & & & & & & 0.042 \\
\hline$<=45$ years & & 139.7 & $(110.2-$ & 169.2) & 88 & $(56.7-$ & 119.3) & \\
\hline 46 to 55 & & 184.4 & $(161.7-$ & 207.1) & 125 & (98.4- & 151.6) & \\
\hline$>=56$ & & 186.1 & $(165.2-$ & 207.1) & 137 & $(103.4-$ & 170.6) & \\
\hline Sex & & & & & & & & 0.221 \\
\hline Female & & 152.5 & $(110.7-$ & 194.3) & 99 & $(62.8-$ & 135.2) & \\
\hline Male & & 179.9 & $(165.3-$ & 194.5) & 119 & $(101.0-$ & 137.0) & \\
\hline \multicolumn{9}{|l|}{ Previous medication } \\
\hline \multirow[t]{2}{*}{ Previous sedative (N03AE N05BA-BB-BX-CD-CF-CM) } & SI & 189.6 & $(162.7-$ & 216.6) & 126 & $(80.3-$ & 171.7) & 0.35 \\
\hline & No & 172.4 & $(156.5-$ & 188.4) & 112 & (93.5- & 130.5) & \\
\hline \multirow[t]{2}{*}{ Sedative in previous 12 months (N03AE N05BA-BB-BX-CD-CF-CM) } & $\mathrm{Si}$ & 208.4 & $(173.8-$ & 243.0) & 160 & $(84.5-$ & 235.5) & 0.045 \\
\hline & No & 169.7 & (154.9- & 184.5) & 111 & (95.1- & 126.9) & \\
\hline \multirow[t]{2}{*}{ Previous antidepressants: GT=N06A / N06B / N06C } & $\mathrm{Si}$ & 181.3 & $(146.5-$ & 216.1) & 137 & (90.2- & 183.8) & 0.577 \\
\hline & No & 176.5 & $(161.5-$ & 191.4) & 111 & (93.6- & 128.4) & \\
\hline \multirow[t]{2}{*}{ Antidepressants in previous 12 months : GT=N06A / N06B / N06C } & $\mathrm{Si}$ & 228.9 & $(174.0-$ & 283.8) & 191 & $(105.3-$ & 276.7) & 0.022 \\
\hline & No & 172.8 & (158.7- & 186.9) & 110 & (92.7- & 127.3) & \\
\hline \multicolumn{9}{|l|}{ Subsequent medication (12 months) } \\
\hline \multirow[t]{2}{*}{ Sedatives } & $\mathrm{Si}$ & 199.3 & $(175.6-$ & 223.0) & 150 & $(113.5-$ & 186.5) & 0.015 \\
\hline & No & 164.2 & (147.5- & 180.9) & 103 & (88.9- & 117.1) & \\
\hline \multirow[t]{2}{*}{ Antidepressants } & $\mathrm{Si}$ & 240.2 & (199.3- & 281.1) & 196 & (133.0- & 259.0) & $<0.001$ \\
\hline & No & 165.9 & (151.6- & 180.2) & 108 & (93.1- & 122.9) & \\
\hline
\end{tabular}

Table 3: Estimation of Hazard Ratio (HR) of return to work in relation to the variables analysed using the Cox regression model

\begin{tabular}{|c|c|c|c|c|c|}
\hline \multicolumn{2}{|l|}{ Variable } & \multirow[b]{2}{*}{$\mathrm{HR}^{*}$} & \multicolumn{2}{|c|}{$95 \% \mathrm{Cl}$} & \multirow[b]{2}{*}{$p$-value } \\
\hline & Category & & (Lower level - & Upper level) & \\
\hline \multicolumn{6}{|l|}{ Sex } \\
\hline & Female & 1.36 & $(1.00-$ & 1.86) & 0,051 \\
\hline \multicolumn{2}{|c|}{ Age in years } & 0.99 & $(0.97-$ & 1.00) & 0.025 \\
\hline \multicolumn{3}{|c|}{ Diagnosis (Ref: Chronic ischemic heart disease) } & & & $<0.001$ \\
\hline & Angina (CIE10:I20) & 1.49 & $(1.12-$ & 1.98) & 0.006 \\
\hline & Acute myocardial infarct (CIE10:I21) & 0.91 & (0.71- & 1.15) & 0.421 \\
\hline \multicolumn{6}{|c|}{ Medication previous 12 month (Ref: None) } \\
\hline & Sedative (N03AE N05BA-BB-BX-CD-CF-CM) & 0.88 & $(1.08-$ & $0.72)$ & 0.217 \\
\hline & Antidepressants GT=N06A / N06B / N06C & 0.64 & $(0.84-$ & $0.48)$ & 0.002 \\
\hline
\end{tabular}

\section{Conclusions}

The mean duration of sick leave due to ischemic heart disease in our health region was almost six months. Consumption of psychotropic medication doubled after the event. Older age, suffering an acute myocardial infarction and taking antidepressants were associated with a longer sick leave period ${ }^{1}$.

\section{Conflict of interest statement}

The authors declare that they have no competing interests.

\section{References}

1. Català N, Serna C, Real J, Yuguero O, Galván L. Assessment of the length of sick leave in patients with ischemic heart disease. BMC cardiovascular disorders. 2017; 17(1): 17-32. 
2. Fernández J, López SE. Carga económica y social de la enfermedad coronaria. Rev Esp Cardiol Supl. 2013; 13(B):42-7.

3. Baena JM, del Val JL, Tomàs J, Martínez JL, Martín R, González I, et al. Epidemiología de las enfermedades cardiovasculares y factores de riesgo en atención primaria. RevEspCardiol. 2005;58:367-73

4. Sicras A, Fernández J, Navarro R, Martín I, Varela C. Morbimortalidad y consumo de recursos asociados tras síndrome coronario agudo en una población española. Revista clínica española. 2011; 211(11):560-71.

5. Oliva J, Lobo F, López J, Duque B, Osuna R. Costes no sanitarios ocasionados por las enfermedades isquémicas del corazón en España. 2004. España: Ministerio de Industria, Comercio y Turismo

6. López J, Rodríguez A, Fernández S. Los principales problemas de salud: Cardiopatía isquémica. AMF: Actualización en Medicina de Familia. 2011;7(6):304-15.

7. Carrera T, Puente E. Cardiopatía Isquémica: Protocolo de estudio en contingencia profesional en Andalucía. http://www. instituto-de-cienciasforenses.com/app/download/9527389/ MME+2011++CARDIOPATIA +ISQUEMICA++word+def.pdf.

8. Jiménez M, Mata C, Bascuas J, Abad E, García AI, Rubio E. Reincorporación laboral del trabajador coronario: criterios objetivos para un cambio de puesto de trabajo. Trauma. 2008;19(3):165-70.

9. Gutiérrez J, Vacas M, Lobato A, Llorca J, Prieto JA, Domenech J, et al. Impacto del infarto de miocardio en la situación laboral de los pacientes. Rev Esp Cardiol. 1999;52:556-62.

10. Tiempos estándar de Incapacidad Temporal. España: Instituto Nacional de la Seguridad Social. 2a edición. http://www.abc.es/ gestordocumental/uploads/economia/Tiempos\%20estandar\%20 incapacidad\%20temporal.pdf.

11. Perk J, Alexanderson K. Sick leave due to coronary artery disease or stroke. Scand J Public Health. 2004;32(63 suppl):181-206. 